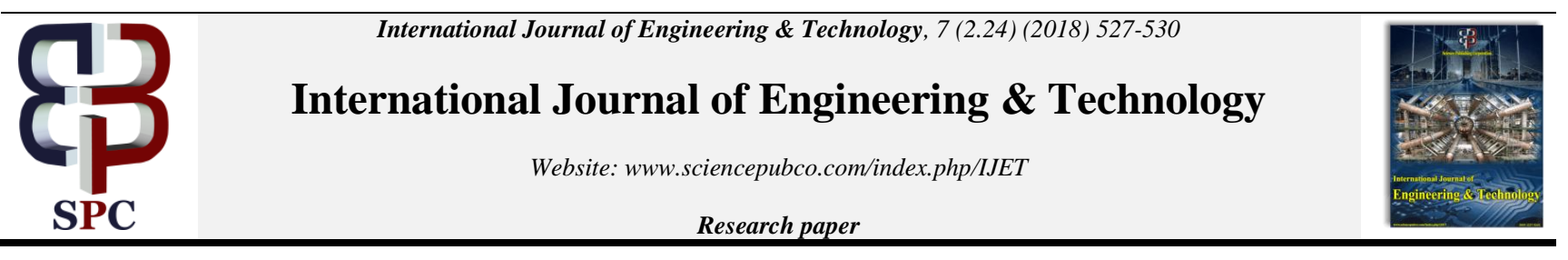

\title{
Driver Assistance System using in-vehicle Traffic Lights and Signs
}

\author{
Vaibhav Jain ${ }^{1 *}$ Tanay $^{2 *}$, Saransh Gangele ${ }^{3 *}$, K. Kalimuthu ${ }^{4 *}$ \\ ${ }^{1,2,3}$ Student, ${ }^{4}$ Associate Professor, SRM Institute of Science and Technology, \\ *Corresponding Author E-mail: vaibhavjain38@gmail.com
}

\begin{abstract}
In recent years, with the advancement of vehicular communication, it is possible to detect various road signs and provide traffic light information to the driver inside the vehicle with the application of heads-up display (HUD). It detects road signs, does basic classifications and accordingly directs the driver to slow down or stop the vehicle. The vehicle's heads-up display keeps the driver focused by providing road warnings, speed limit, traffic signals and some vital navigation information in the driver's line of sight(LOS). This system has 4 phases, Image recognition, wireless communication, obstacle detection and driver mechanism. This system aims to create a prototype of a smart driver assistance system which provides better road traffic and driver's safety in countries with high traffic congestion where fully automated vehicles cannot function effectively. This system can be easily implemented in real time scenarios to reduce accidents and enhance the convenience of driving.
\end{abstract}

Keywords: Driver Mechanism, Heads-up display, Image Recognition, Wireless Communication, Vehicular Communication

\section{Introduction}

According to the International car sales statistical report, around 79 million cars have been sold in the year 2017 and nearly 81.6 million cars are expected to be sold by the end of 2018. These statistics indicate the increasing number of vehicles on the road which results in high traffic con-gestion in many countries and the number of vehicles will only continue to grow rapidly in the upcoming years. This high surge in demand for vehicles has also resulted in an increasing number of road accidents which puts not only the drivers lives at risk but also the pedestrians lives. This paper proposes a prototype of a Driver Assistance System utilizing heads-up display to counter this problem effectively. The prototype would initially detect all the road signs and signals using a camera. The information will then be pro-cessed and transmitted via a zigbee module to the Arduino microcontroller. The data received will then be displayed on the heads-up display in front of the driver. Additionally, an ultrasonic sensor is used for obstacle detection whose data is also displayed on the HUD. When the vehicle exceeds a preset threshold of distance, it starts to slow down or comes to rest if required to avoid collision.

\section{Literature Survey}

There are many conventional ways already existing in the previous projects using which a driver assistance system can be made one such way is using Bluetooth for communication process [1], [5], but in our proposed system, ZigBee is pre-ferred for wireless communication process because ZigBee has more power profile, less latency, high data rate and much higher range than Bluetooth. Also, it is less complex than Bluetooth. The entire algorithm for object detection is based on image processing using MATLAB [6].
A camera is used as an image acquisition device, Although it is preferred to use a high resolution external USB camera but an inbuilt laptop camera can also be used. For displaying the data or sign, a smartphone [5] or a heads-up display can be used. However, heads-up display has an edge over a smartphone as a smartphone can face connectivity issues during a call and can fail to provide the required information whereas HUD will not face any such issues. Also, HUD has a shorter glance time [2]. Thus, it is capable of displaying signs and instructions improving drivers situational awareness while not increasing the drivers mental task load relative to natural driving [7].

\section{System Model}

Initially, the camera scans for any traffic sign or signal on the road. Once a traffic sign or signal is detected it then compares it with its database and determines which sign it is. The processed information is then sent to the Arduino. The Arduino here acts as an interface between the vehicle and the driver alert system (HUD). The Message is then displayed on the HUD to alert the driver. Simultaneously the Ultrasonic sensor keeps on checking the distance between the nearby vehicles, if the distance crosses a preset threshold value or two vehicles come too close it would either slow the vehicle down or completely stop it. 


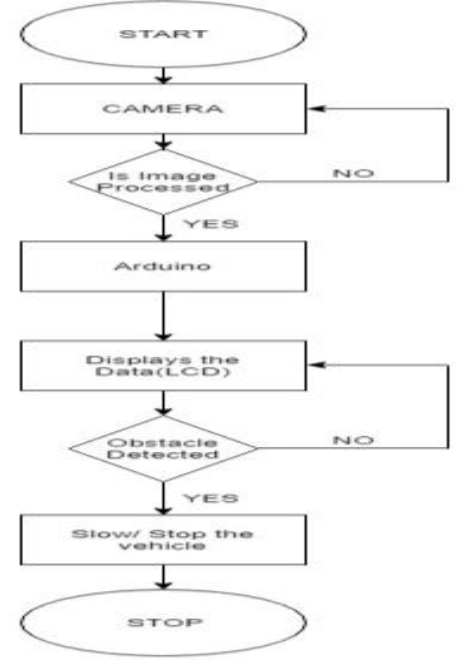

Fig. 1: Flow Chart of the Prototype

\section{System Architecture}

The Overall Architecture of the prototype is described below

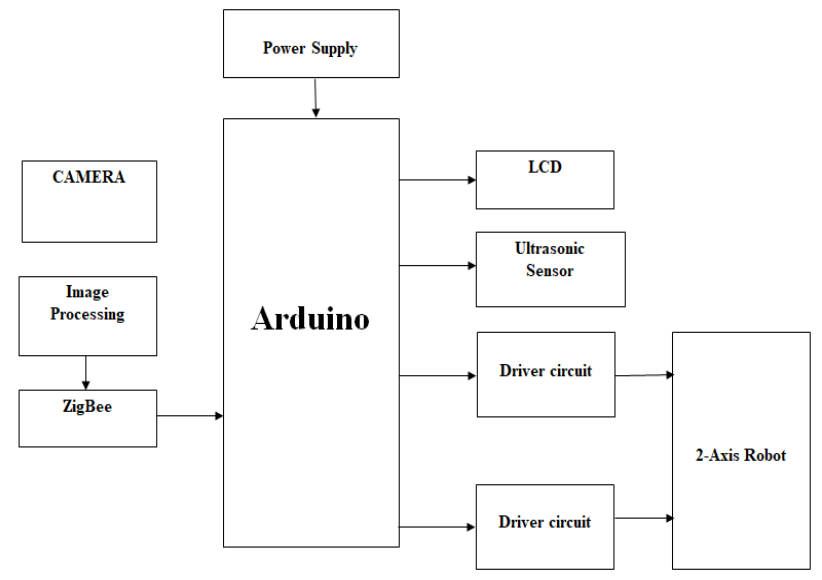

Fig. 2: Block Diagram of the prototype

\subsection{Sign and Signal Detection}

The traffic sign and signal detection is done using image processing. For this prototype MATLAB has been used for the process. The Camera first captures the image. The image is then converted into a 2-D grayscale image. The system is trained for various signs and a database is created. Speeded-Up Robust Features (SURF) algorithm is used along with k-nearest neighbors (k-NN) classifier.

SURF: Speeded Up Robust Features is an algorithm for image recognition, registration and clas-sification. The process falls under the category of fea-ture descriptors which basically works on extracting keypoints from distinct regions of the image and thus can be employed to find similarities between multiple images. This algorithm has three main parts: Interest point detection, local neighbourhood description and matching the image to the pretrained set of images. The SURF descriptors are generally used to recognise and locate objects, people or faces and to extract key points of interests. And then the descriptors are used to match the concerned image with pre-trained sets of different images and thus the matching pair of images can be found. SURF is way faster than other methods of image recognition as it uses descriptors to detect landmarks in the image that can be used to compare the images and find exact match for it.

KNN: kNN or k - nearest neighbour is an image classi-fication strategy that uses the local features of an image rather than looking for similarity between different sets of images. A basic kNN algorithm for image classifi-cation determine an image by searching for $\mathrm{k}$ images in the training set and by performing a class weighted frequency analysis, the $\mathrm{k}$ closest images are determined depending on the similarity measures between entire images. With the application of local features and interest points, $\mathrm{kNN}$ classification system can be altered to consider the similarities between the local features of multiple images rather than training the system for similarities between entire images which can prove to be way more efficient and is far easier to handle. It is basically done in two steps, First: Each local feature is ranked according to the local characteristics of the learning set; Second: The entire image is classified taking into consideration the class assigned to each local feature and the reliability of this classification.

\subsection{Data Transmission}

The converted hexadecimal data is then transferred to the Arduino. The data is transferred serially using a Zigbee module. The transmitter module of the Zigbee is connected to the camera and the receiver module is connected to the Arduino. The data is sent in the form of bits at the speed of $250 \mathrm{kbps}$ which results in minimal delay between the image recognition and the displaying of data.

\subsection{Data Display (HUD)}

The data received are codes in hexadecimal form. Each code has been assigned the same sign or signal name from which it was initially converted. The heads-up display is connected to the Arduino which will display the name of the sign.

\subsection{Obstacle Detection Mechanism}

Obstacle detection Mechanism is used to detect any ob-stacle present near the vehicle. This mechanism not only checks for the Obstacle but also checks if there is any free area available near the vehicle. An Ultrasonic sensor is being used to measure the distance. There is a preset threshold value which when exceeded the vehicle starts to slow and comes to rest if the obstacle is considerably close to the vehicle.

\subsection{Driving Mechanism}

The Driving mechanism takes care of controlling the vehicle if the driver fails to respond to the signals, signs or if there is a vehicle or an obstacle in front of the vehicle. A driver circuit in this case L293D is connected to the vehicle that makes sure that the vehicle slows down or comes to rest in a situation when an obstacle is dangerously close to the vehicle.

\section{Circuit Design}

Below are the schematic diagram and MATLAB simulation of the prototype..

\subsection{Circuit Diagram}

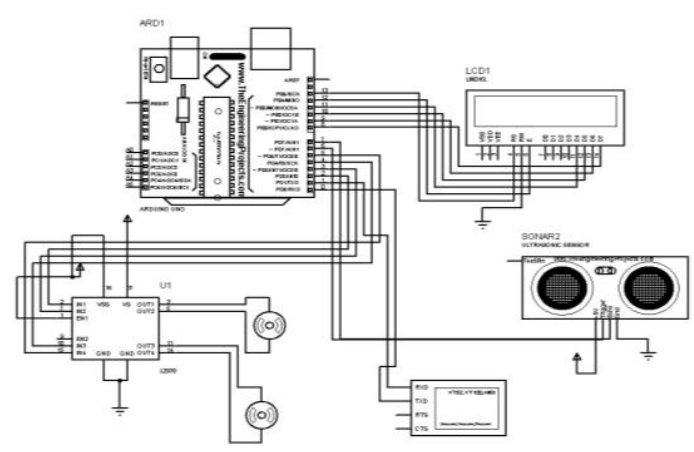

Fig. 3: Schematic Diagram of the Prototype 


\subsection{MATLAB Simulation}

Below show are the simulation of two different signs,Men at Work and School Zone respectively. Fig 4 and Fig 6 shows the image detection in MATLAB and Fig 5 and Fig 7 shows the Output on HUD

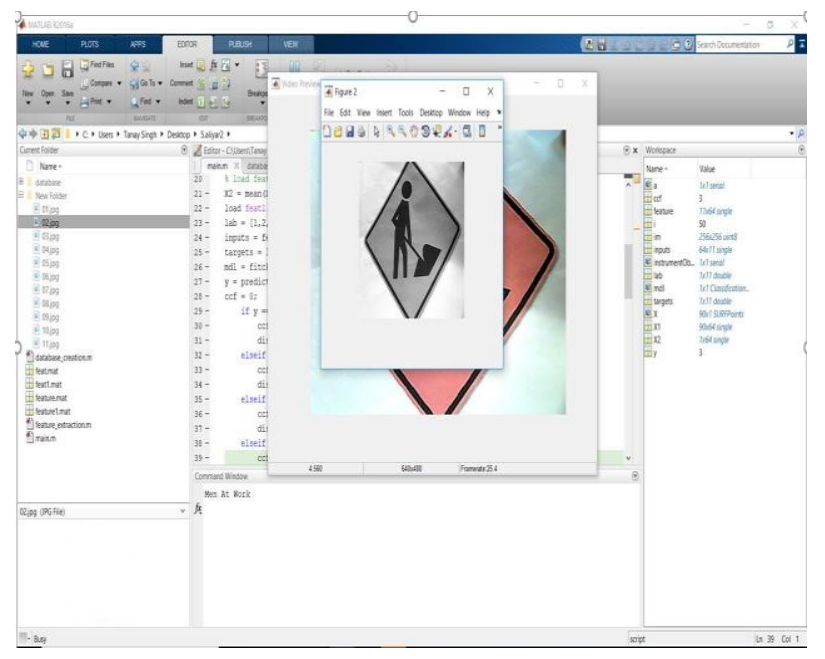

Fig. 4: Image Detection in Matlab(Men at Work)

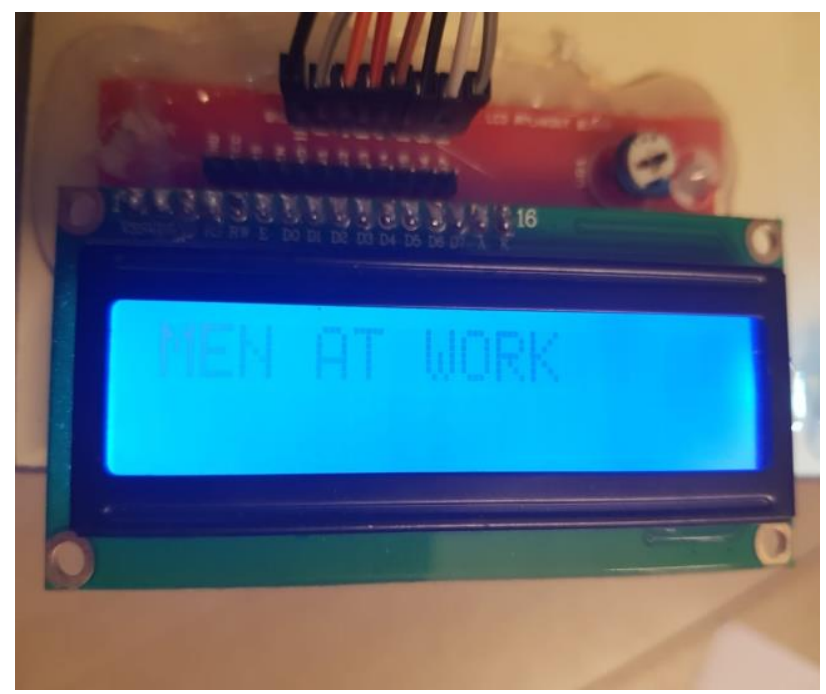

Fig. 5: Data Display in HUD (Men at Work)

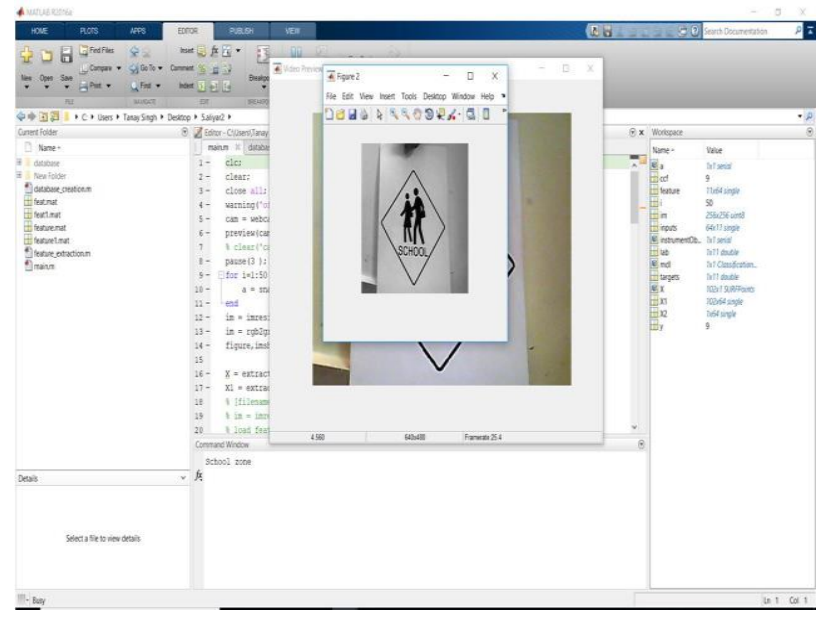

Fig 6: Image Detection in Matlab (School Zone)

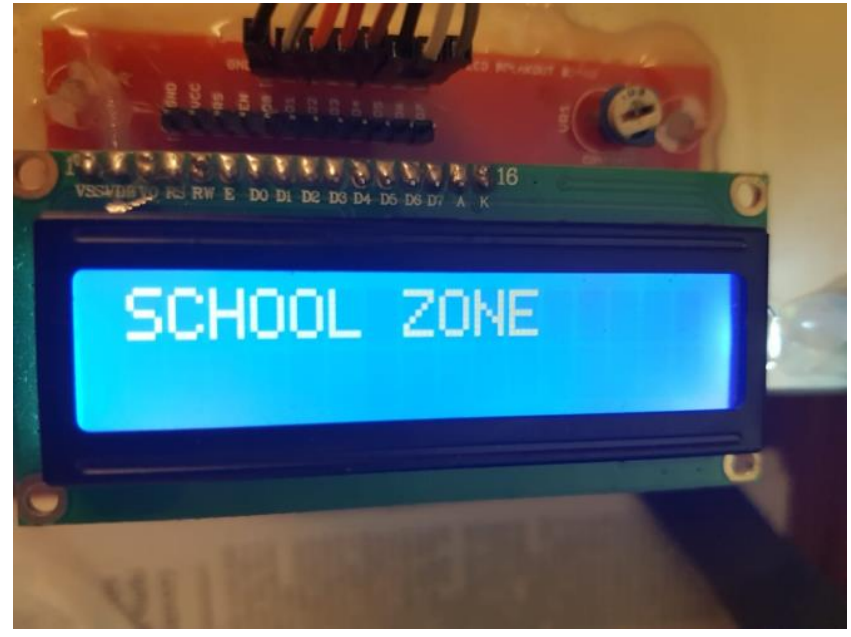

Fig. 7: Data Display in HUD(School Zone)

\section{Hardware Description}

\subsection{Arduino}

It is an open-source electronics platform formed on adapt-able hardware and software. It is used to read multiple kinds of input and produce various outputs. This is achieved by sending a certain set of instructions to the microcontroller present on the board. The board is furnished with multiple sets of digital and analog $\mathrm{I} / \mathrm{O}$ pins that can be interfaced to various kinds of circuits for endless applications. Most Arduino boards are made up of an Atmel 8-bit AVR micro-controller with different amount of flash memory, pins, and features. The Arduino IDE is a cross platform software that runs on Windows, MAC and Linux.

\subsection{Zigbee}

ZigBee is built for sensor and control networks on IEEE 802.15.4 standard for wireless communication. This communication standard is widely deployed in the Physical and MAC layer in order to handle multiple devices at a time at lower data rates. It primarily operates at a frequency of $2.4 \mathrm{GHz}$ and having a data rate of $250 \mathrm{kbps}$ while having an optimum range of 10-100 metres. It can be used for multiple applications in wireless field such as monitoring, automation and maintenance of multiple systems.

\subsection{Ultrasonic Sensor}

An ultrasonic sonic sensor is an electronic device that is used to measure the distance to a distant object by using sound waves. It works by sending out a sound wave at a certain frequency and waiting for the sound wave to bounce back. By measuring the time lapse between generating the sound wave and receiving it again after bouncing back, the distance between the two objects is measured. The ultrasonic sensor has 4 pins, namely VCC, GND, TRIG and ECHO which are used to connect the device to other interfacing circuits.

\subsection{Driver Circuit (L293D)}

L293D is a motor driver IC that can be used to drive a DX motor in either direction. It has a 16 pin IC that controls two DC motor simultaneously in any given direction. It works on the principle of H-bridge that allows voltage to flow in either direction. So an L293D chip has two H-bridge circuits that facilitates the operation of two driver motors. The circuit acts as current amplifier that takes low current control signal and produce a high value of output. This chip can operate in an optimum range of $5 \mathrm{~V}$ to $36 \mathrm{~V}$. 


\section{Software Description}

\subsection{MATLAB}

MATLAB is a high level language for scientific com-puting. It seamlessly helps in integrating visualization and computation in a programmable environment. The problems and necessary solutions are expressed in known mathematical notations. It is basically a programming language based on a desktop environment that expresses matrices and array mathematics precisely.

\subsection{Proteus}

Proteus is an exclusive software tool suite employed for circuit design automation. It is mainly used to create schematics for manufacturing printed circuit boards. The software offers netlist based PCB design with auto place-ment, auto routing and graphical simulation results.

\section{Future Work}

The Proposed project after few modifications can also be used to alert the driver by annotating the map with lights that always flash in case of an emergency [4].The system can further be integrated with a speaker to give audio alerts along with the displaying of data. Further, the system can be modified for audio recognition to perform various other functions. With the help of Machine Learning(ML), the system will be able to predict the next mode of traffic light. There will be two modes, a current mode and a predicted mode. If the image captured is a red light, then the system will be able to predict that the next mode will be a green light in $\mathrm{x}$ seconds [8].Also using ML the proposed system will be able to detect and distinguish between 2 different traffic signal which are at a certain distance from each other and will consider the one closest to it [3].

\section{Conclusion}

This prototype is developed to alert the driver about the obstacles and the traffic signs and signals if driver fails to notice them. The proposed model is designed to make driving more safe and convenient in countries where there is a problem of congested traffic as completely automated cars will not be able to function properly in such countries. The system does not aim to replace the driver with an automated system, rather provide assistance to the driver for better road awareness.

\section{References}

[1] Stweart A Birrell, Mark Fowkes and Paul A Jennings. "Effect of using an in-vehicle smart driving aid on real-world driver performance", IEEE Transactions on Intelligent Transportation System, 15(4):1801-1810,2014

[2] Siddhant Chouksey and Sumedha Sirsikar. "A prototype of low cost heads up display for automobiles navigation system". In Computing, Analytics and Security Trends (CAST), International Conference on, pages 205-210. IEEE, 2016

[3] Moises Diaz-Cabrera, Pietro Cerri, and Javier Sanchez-Medina, "Sus-pended traffic lights detection and distance estimation using color features". In In Intelligent Transportation Systems (ITSC), 2012 15th International IEEE Conference on, pages 1315-1320. IEEE, 2012.

[4] Nathaniel Fairfield and Chris Urmson. "Traffic light mapping and detection". In Robotics and Automation (ICRA), 2011 IEEE International Conference on, pages 5421-5426. IEEE, 2011.

[5] Ching-Hao Lai and Chia-Chen Yu, "An efficient real-time traffic sign recognition system for intelligent vehicles with smart phones". In Technologies and Applications of Artificial Intelligence (TAAI), 2010 International Conference on, pages 195-202. IEEE, 2010.

[6] Panth Shah and Tithi Vyas, "Interfacing of matlab with arduino for object detection algorithm implementation using serial communica- tion". International Journal of Engineering Research \& Technology(IJERT) IJERT ISSN, pages 2278-0181, 2014.

[7] Geva Vashitz, David Shinar, and Yuval Blum, "In-vehicle information systems to improve traffic safety in road tunnels". Transportation Research Part F: Traffic Psychology and Behaviour, 11(1):61-74, 2008.

[8] Bo Yang, Rencheng Zheng, Yuandong Yin, Shigeyuki Yamabe, and Kimihiko Nakano, "Analysis of influence on driver behaviour while using in-vehicle traffic lights with application of head-up display” IET Intelligent Transport Systems, 10(5):347-353, 2016. 